\title{
Magnetic Excitations in the Spin-1 Anisotropic Heisenberg Antiferromagnetic Chain System $\mathrm{NiCl}_{2}-4 \mathrm{SC}\left(\mathrm{NH}_{2}\right)_{2}$
}

\author{
S. A. Zvyagin, ${ }^{1}$ J. Wosnitza, ${ }^{1}$ C. D. Batista, ${ }^{2}$ M. Tsukamoto, ${ }^{3}$ N. Kawashima, ${ }^{3}$ \\ J. Krzystek, ${ }^{4}$ V. S. Zapf, ${ }^{5}$ M. Jaime, ${ }^{5}$ N. F. Oliveira, Jr., ${ }^{6}$ and A. Paduan-Filho ${ }^{6}$ \\ ${ }^{I}$ Dresden High Magnetic Field Laboratory (HLD), Forschungszentrum Dresden - Rossendorf, 01314 Dresden, Germany \\ ${ }^{2}$ Theoretical Division, Los Alamos National Laboratory, Los Alamos, NM 87545, USA \\ ${ }^{3}$ Institute for Solid State Physics, University of Tokyo, Kashiwa, Chiba 227-8581, Japan \\ ${ }^{4}$ National High Magnetic Field Laboratory, Florida State University, Tallahassee, FL 32310, USA \\ ${ }^{5}$ National High Magnetic Field Laboratory, Los Alamos National Laboratory, MS-E536, Los Alamos, NM 87545, USA \\ ${ }^{6}$ Instituto de Fisica, Universidade de Sao Paulo, 05315-970 Sao Paulo, Brazil
}

\begin{abstract}
$\mathrm{NiCl}_{2}-4 \mathrm{SC}\left(\mathrm{NH}_{2}\right)_{2}$ (DTN) is a quantum $S=1$ chain system with strong easy-pane anisotropy and a new candidate for the Bose-Einstein condensation of the spin degrees of freedom. ESR studies of magnetic excitations in DTN in fields up to $25 \mathrm{~T}$ are presented. Based on analysis of the single-magnon excitation mode in the high-field spin-polarized phase and previous experimental results [Phys. Rev. Lett. 96, 077204 (2006)], a revised set of spin-Hamiltonian parameters is obtained. Our results yield $D=8.9 \mathrm{~K}, J_{c}=2.2 \mathrm{~K}$, and $J_{a, b}=0.18 \mathrm{~K}$ for the anisotropy, intrachain, and interchain exchange interactions, respectively. These values are used to calculate the antiferromagnetic phase boundary, magnetization and the frequency-field dependence of two-magnon bound-state excitations predicted by theory and observed in DTN for the first time. Excellent quantitative agreement with experimental data is obtained.
\end{abstract}

PACS numbers: 75.40.Gb, 76.30.-v, 75.10.Jm

Antiferromagnetic (AFM) quantum spin-1 chains have been the subject of intensive theoretical and experimental studies, fostered especially by the Haldane conjecture [1]. Due to quantum fluctuations, an isotropic spin- 1 chain has a spin-singlet ground state separated from the first excited state by a gap $\Delta \sim 0.41 J[2]$, where $J$ is the exchange interaction. As shown by Golinelli et al. [3], the presence of a strong easy-plane anisotropy $D$ can significantly modify the excitation spectrum, so that the gap size is not determined by the strength of the AFM quantum fluctuations exclusively, but depends on the dimensionless parameter $\rho=D / J$. The Haldane phase is predicted to survive up to $\rho_{c}=0.93$ [4], where the system undergoes a quantum phase transition. For $\rho>\rho_{c}$ the gap reopens, but its origin is dominated by the anisotropy $D$, and the system is in the so-called large- $D$ regime. While the underlying physics of Haldane chains is fairly well understood, relatively little is known about the magnetic properties (and particularly the elementary excitation spectrum) of nonHaldane $S=1$ AFM chains in the large- $D$ phase. Intense theoretical work and numerous predictions $[3,4,4,5,6,6,7,8,6,9,10]$ make the experimental investigation of large- $D$ spin- 1 chains a topical problem in low-dimensional magnetism.

Recently, weakly-coupled spin-1 chains have attracted renewed interest due to their possible relevance to the fieldinduced Bose-Einstein condensation (BEC) of magnons. When the field $H$, applied perpendicular to the easy plane, exceeds a critical value $H_{c 1}$ (defined at $T=0$ ), the gap closes and the system undergoes a transition into an $X Y$-like AFM phase with a finite magnetization and AFM magnon excitations. If the spin Hamiltonian has axial symmetry with respect to the applied field, the AFM ordering can be described as BEC of magnons by mapping the spin- 1 system into a gas of semi-hard-core bosons [11]. The applied field plays the role of a chemical potential, changing the boson population. In accordance with mean-field BEC theory [12, 13, 14], the phasediagram boundary for a three-dimensional system should obey a power-law dependence, $H-H_{c 1} \sim T_{c}^{3 / 2}$, as $T \rightarrow 0$. Above the upper critical field, $H_{c 2}$ (defined at $T=0$ ), the system is in a fully spin-polarized (FSP) phase, and the excitation spectrum is formed by gapped magnons.

The compound $\mathrm{NiCl}_{2}-4 \mathrm{SC}\left(\mathrm{NH}_{2}\right)_{2}$ (dichloro-tetrakis thiourea-nickel(II), known as DTN), is a new candidate for studying the BEC phenomenon in magnetic fields. It has a tetragonal crystal structure, space group $I 4$, with two molecules in the unit cell [15, 16]. The anisotropy, intra- and inter-chain exchange parameters, $D=8.12 \mathrm{~K}, J_{c}=1.74 \mathrm{~K}$, and $J_{a, b}=0.17 \mathrm{~K}$, respectively, were obtained from a fit of zero-field inelastic-neutron-scattering data [17] using generalized spin-wave theory [18]. It was shown that the $\mathrm{Ni}$ spins are strongly coupled along the tetragonal axis, making DTN a system of weakly interacting $S=1$ chains with single-ion anisotropy larger than the intra-chain exchange coupling. Recently, it was proposed [16, 17] that the field-induced low-temperature transition of DTN to the AFM-ordered state can be interpreted as a BEC of magnons, with values of $H_{c 1}=2.1 \mathrm{~T}$ and $H_{c 2}=12.6 \mathrm{~T}$ for the lower and upper critical fields, respectively. Although the observed overall picture is consistent with the BEC scenario, some questions remain. One of the unsolved problems is a pronounced (about $2 \mathrm{~T}$ ) disagreement between the value for the upper critical field obtained experimentally and the predicted one $\left(H_{c 2}=10.85 \mathrm{~T}\right)[17]$. This discrepancy is rather puzzling, particularly in light of the excellent agreement for $H_{c 1}$.

In this Letter, we report electron spin resonance (ESR) studies of the elementary excitation spectrum in DTN in magnetic fields up to $25 \mathrm{~T}\left(\sim 2 \mathrm{H}_{c 2}\right)$. A distinct advantage of the high- 


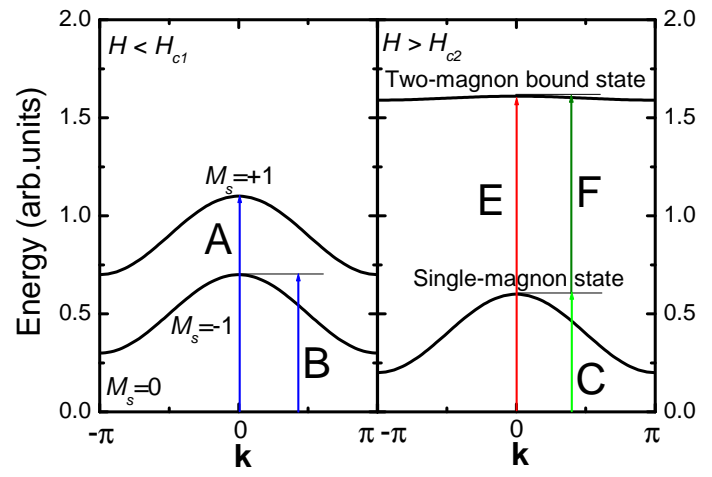

FIG. 1: (color online) A schematic view of the magnetic-excitation dispersion in an $S=1$ Heisenberg chain with strong easy-plane $(D>0)$ anisotropy for two arbitrary fields, $H<H_{c 1}$ (left) and $H>H_{c 2}$ (right), with a magnetic field applied along the principal axis $z$. Note that the ESR transitions denoted by A, B, C, E, and F occur at $\mathbf{k}=0$. Two-particle continua predicted for both regions are not shown for simplicity.

field approach for determining the spin-Hamiltonian parameters of spin-1 AFM chains is the availability of exact theoretical expressions for the spin-polarized phase. Analysis of the high-field single-magnon branch has allowed us to obtain the precise value for the anisotropy parameter, $D=8.9 \mathrm{~K}$. Using results of recent thermodynamic and neutron-scattering measurements [16, 17], we were able to refine the exchange parameters, obtaining $J_{c}=2.2 \mathrm{~K}, J_{a, b}=0.18 \mathrm{~K}$ for intraand inter-chain exchange interactions, respectively. In addition, we present magnetocaloric-effect and low-temperature magnetization data allowing us to check the obtained set of parameters. These values agree well with those obtained by fitting the AFM phase boundary and the magnetization with results of quantum Monte Carlo simulations, and nicely reproduce both critical fields, $H_{c 1}=2.1 \mathrm{~T}$ and $H_{c 2}=12.6 \mathrm{~T}$. Furthermore, we report on the first direct and reliable observation of the two-magnon bound states in spin-1 AFM chain system with strong easy-plane anisotropy, predicted by theory [9] for the high-field spin-polarized phase. Using the obtained parameters of the spin-Hamiltonian we were able to calculate frequency-field dependences of the two-magnon bound state excitations. Excellent quantitative agreement between the theoretical predictions and experiment is obtained.

The investigation of the magnetic excitation spectra in single-crystalline DTN samples was done using a tunablefrequency submillimeter-wave ESR spectrometer [19] with external field $H$ applied along the tetragonal $c$ axis.

In sufficiently small fields, a spin-1 $\mathrm{Ni}$ (II) system is in the quantum-paramagnetic (QPM) phase, having the $M_{s}=0$ ground state. The excitation spectrum is gapped and determined by the $\Delta M_{s}= \pm 1$ transitions. For fields $H$ applied perpendicular to the easy plane, the Hamiltonian can be writ- ten as

$$
\mathcal{H}=\sum_{\mathbf{j}, \nu} J_{\nu} \mathbf{S}_{\mathbf{j}} \cdot \mathbf{S}_{\mathbf{j}+e_{\nu}}+\sum_{\mathbf{j}}\left[D\left(S_{\mathbf{j}}^{z}\right)^{2}+g \mu_{B} H S_{\mathbf{j}}^{z}\right]
$$

where $\nu=\{a, b, c\}$. A corresponding dispersion of low-field magnetic excitations is shown schematically in Fig. 1 (left). Then, the frequency-field dependence of the $\Delta M_{s}= \pm 1$ ESR transitions can be written as $\omega^{A / B}=\Delta \pm g \mu_{B} H$, where $\Delta$ corresponds to the energy gap at $\mathbf{k}=0$ and $H=0$. These transitions were observed in our experiments and are denoted in Fig. 2]by circles [20]. The zero-field splitting measured directly yields $\Delta=269 \mathrm{GHz}$, which agrees quite well with results of neutron-scattering measurements, $\Delta_{\mathbf{k}=0}=1.1$ $\mathrm{meV}$ [17]. No splitting within the excited doublet in zero field was observed, which indicates the absence of in-plane anisotropy. From the slope of the frequency-field dependences of the branches A and B, the $g$ factor was determined directly, $g=2.26[21]$.

As mentioned before, when the magnetic field exceeds the upper critical field, $H_{c 2}$, the system is in the fully spinpolarized state (a corresponding dispersion of low-energy magnetic excitations is shown schematically in Fig.1(right)). Excitations from the ground state to the single-magnon state have been observed in our experiments and are denoted by squares in Fig. 2 (the resonance C). These excitations correspond to a single-spin flip from the $S_{z}=-1$ to $S_{z}=0$ state and are uniformly delocalized over the entire lattice with a well-defined momentum $\mathbf{k}$. The $S_{z}=0$ state propagates along the lattice as a free quasiparticle with hopping $J_{\nu}$ along the $\nu$ direction $(\nu=\{a, b, c\})$, that arises from the transverse

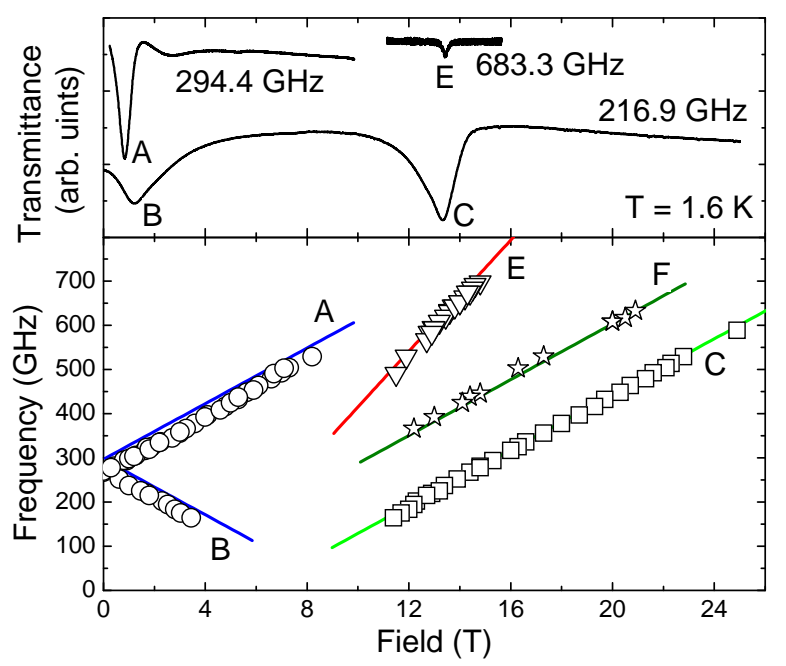

FIG. 2: (color online) Top: Typical ESR transmission spectra in DTN, taken at frequencies of 216.9, 294.4, and $683.3 \mathrm{GHz}$ at $T=$ $1.6 \mathrm{~K}, H \| c$. Bottom: Frequency-field dependence of magnetic excitations in DTN, taken at temperatures of $1.6 \mathrm{~K}$ (the modes A, B, C, and $\mathrm{E}$ ) and $4.3 \mathrm{~K}$ (the mode $\mathrm{F}$ ), $H \| c$. Symbols denote the experimental results, and lines correspond to results of calculations (see text for details). 


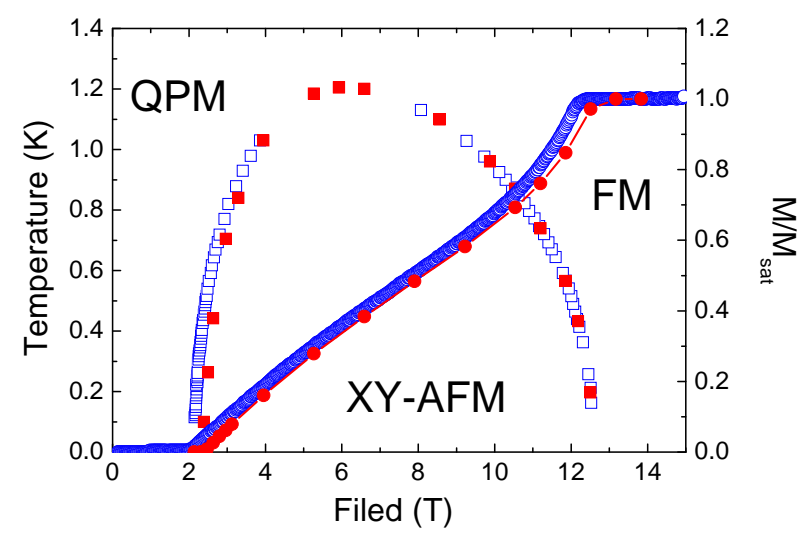

FIG. 3: (color online) Temperature-field diagram of the AFMordered phase obtained from magnetocaloric-effect measurements (opened squares), and the magnetization data taken at $T=16 \mathrm{mK}$ (opened circles), $H \| c$. Results of the quantum Monte Carlo calculations of the phase-diagram boundaries and the magnetization using the set of parameters obtained as described in text are denoted by closed squares and circles, respectively.

part of the Heisenberg interaction. There are also diagonal energy gains of $-2\left(J_{c}+2 J_{a}\right)$ due to the Ising part, and $-D$ due to the single-ion anisotropy. The diagonal energy cost comes from the Zeeman interaction $g \mu_{B} H$. Then, the single-magnon excitation dispersion can be calculated exactly:

$$
\begin{array}{r}
\omega(\mathbf{k})=g \mu_{B} H-D-2\left(J_{c}+2 J_{a}\right)+2\left(J_{c} \cos k_{z}+\right. \\
\left.J_{a} \cos k_{x}+J_{b} \cos k_{y}\right) .
\end{array}
$$

The ESR transitions taking place at $\mathbf{k}=0$ have the frequency $\omega_{C}=g \mu_{B} H-D$. The best fit of the ESR data denoted by squares in Fig. 2 reveals $D=8.9 \mathrm{~K}$ for the anisotropy constant. From the exact expression for $H_{c 2}$, given as [17]

$$
H_{c 2}=\frac{1}{g \mu_{B}}\left(D+4 \sum_{\eta} J_{\eta}\right)
$$

and using $H_{c 2}=12.6 \mathrm{~T}\left[16\right.$, 17] we obtain $\sum_{\eta} J_{\eta}=$ $J_{c}+2 J_{a}=2.557 \mathrm{~K}$. The zero-field dispersion of magnetic excitations calculated using neutron-scattering data [17] yields $J_{a} / J_{c}=0.082$. Thus, in addition to the anisotropy constant $D=8.9 \mathrm{~K}$, all three exchange parameters, $J_{c}=2.2 \mathrm{~K}$ and $J_{a}=J_{b}=0.18 \mathrm{~K}$, can be calculated quite precisely.

The phase boundary (obtained from magnetocaloric-effect measurements) and the field dependence of the magnetization at $T=16 \mathrm{mK}$ were computed for the obtained set of parameters by means of a quantum Monte Carlo simulations for a finite lattice of $L^{3}$ sites, $L=16$. Fig. 3 shows a very good agreement between the calculated (closed symbols) and experimental (opened symbols) data.

In addition to ordinary single-magnon states and twomagnon continuum, the theory [9] predicts the existence of two-magnon bound states (sometimes referred to as singleion bound states [5]). The physical picture of the two-magnon bound-state excitations corresponds to a double-spin-flip transition from $S_{z}=-1$ to $S_{z}=+1$. The transverse part of the Heisenberg term of $\mathcal{H}$ mixes this state with the one that has a pair of $S_{z}=0$ states. Since the diagonal energy difference between these two states, $2 D$, is much bigger than $J_{c}$ and $J_{a}$ (associated with hopping in the $c$ and $a$ directions), the distance between the two $S_{z}=0$ sites remains finite, giving rise to a two-magnon bound state. The two-magnon bound states appear to be a specific feature of anisotropic spin-1 Heisenberg systems. It is worth mentioning that the two-magnon bound states were already predicted in 1970 by Silberglitt and Torrance [5] for Heisenberg ferromagnets with single-ion anisotropy. Later on, this subject attracted a great deal of attention due to its potential relevance to the intrinsic localized spin modes in anisotropic ferromagnets [22] and antiferromagnets [23]. It was suggested [9] that the two-magnon bound states should make a distinct contribution to the excitation spectrum of $\mathrm{S}=1$ large- $D$ AFM chains above the upper critical field $H_{c 2}$ and that their effect can be unambiguously identified by ESR measurements. A signature of two-magnon bound states was obtained by means of high-field ESR in the spin- 1 chain compound $\mathrm{Ni}\left(\mathrm{C}_{2} \mathrm{H}_{8} \mathrm{~N}_{2}\right)_{2} \mathrm{Ni}(\mathrm{CN})_{4}$ (known as NENC) [24]. A broad absorption was detected in the highfield spin-polarized phase. Based on analysis of the temperature dependence of the ESR intensity, this feature was interpreted as transitions from the single-magnon to two-magnon bound states. However, a closer look at ESR spectra in NENC uncovered a more complicated structure than predicted (including, for instance, two non-equivalent $\mathrm{Ni}$ sites and, most likely, a finite in-plane anisotropy), which made the accurate quantitative comparison with the theory difficult.

Here we report on the first observation of transitions from the ground state to two-magnon bound state in a spin-1 AFM chain system with strong easy-plane anisotropy in the highfield FSP phase. The corresponding excitations are denoted by triangles in Fig. 2] bottom (the resonance E, Fig. 2(top)). The frequency-field dependence of the ground-state two-magnon bound-state excitations can be calculated exactly using the set of parameters obtained as described above. Results of corresponding calculations are shown in Fig. 2 (bottom) by the line E. One more resonance absorption was observed at higher temperatures (which indicates transitions within excited states). The corresponding data obtained at $T=4.3 \mathrm{~K}$ are denoted in Fig. 2 (bottom) by stars. This ESR mode corresponds to transitions from the single-magnon to two-magnon bound states (Fig. 1 (right)), which occur at $\mathbf{k}=0$. The frequency-field dependence of these transitions can be calculated, using the expression $\omega_{F}=\omega_{E}-\omega_{C}$ (where $\omega_{E}$ and $\omega_{C}$ are the excitation frequencies for the modes $\mathrm{E}$ and $\mathrm{C}$, respectively), and is denoted in Fig. 2 2 by the line F. In both cases, excellent agreement with experimental data was achieved, which shows that our model actually works fairly well up to $T \sim J$.

Let us now discuss the low-field quantum-paramagnetic phase. As mentioned before, in this phase the system has the $M_{s}=0$ ground state and a gapped excitation spectrum formed by $\Delta M_{s}= \pm 1$ transitions. The obtained set of spin- 
Hamiltonian parameters can be used to calculate the lower critical field, employing the spin-wave theoretical approach [18]:

$$
H_{c 1}=\frac{1}{g \mu_{B}} \sqrt{\mu^{2}-4 s^{2} \mu \sum_{\eta} J_{\eta}} .
$$

The parameters $\mu=10.3$ and $s^{2}=0.92$ can be obtained from the self-consistent equations:

$$
D=\mu\left(1+\frac{1}{N_{s}} \sum_{\mathbf{k}} \frac{\gamma_{\mathbf{k}}}{\omega_{\mathbf{k}}}\right)
$$

and

$$
s^{2}=2-\frac{1}{N_{s}} \sum_{\mathbf{k}} \frac{\mu+s^{2} \gamma_{\mathbf{k}}}{\omega_{\mathbf{k}}}
$$

where $\gamma_{\mathbf{k}}=2 \sum_{\nu} J_{\nu} \cos k_{\nu}$ and $\omega_{\mathbf{k}}=\omega_{\mathbf{k}}^{A / B}(H=0)$. It is important to mention, that the disagreement between the calculated $\left(H_{c 1}=2 \mathrm{~T}\right)$ and experimental $\left(H_{c 1}=2.1 \mathrm{~T}\right)$ values is due to limitations of the spin-wave theory applied to quasione-dimensional spin systems. Using the spin-wave theory [18] and the set of parameters obtained as described above, the frequency-field dependence of the ESR transitions can be calculated using the expression [17]

$$
\omega^{A / B}=\sqrt{\mu^{2}+4 s^{2} \mu \sum_{\eta} J_{\eta}} \pm g \mu_{B} H .
$$

Eq. (7) predicts a value of $H_{c 1}=2 \mathrm{~T}$, that is in fact in relatively good agreement with the experimental value $H_{c 1}=2.1$ $\mathrm{T}$. The results of the calculations are presented by the lines A and B in Fig. 2 (bottom) together with experimental data denoted by circles. The agreement in this case is reasonably good, considering that the quasi-one-dimensional nature of the system causes pronounced quantum fluctuations of the AFM-order parameter in the low-field QPM phase.

In summary, a systematic ESR study of the magnetic excitations in DTN, an $S=1$ Heisenberg AFM chain material in the large-D regime, has been presented. Investigation of the high-field magnon excitations allowed us to obtain a reliable set of the spin-Hamiltonian parameters, which was employed to calculate the ESR spectrum in a broad range of magnetic fields and frequencies. These values agree very well with the ones obtained from fitting the AFM-phase boundary and low-temperature magnetization of DTN with results of quantum Monte Carlo simulations, including both critical fields. The parameters were used to calculate the frequency-field dependence of two-magnon bound-state excitations, predicted by theory and observed in DTN for the first time. Excellent agreement between the theory and experiment was obtained.
Acknowledgments.- The authors express their sincere thanks to A.K. Kolezhuk, A. Orendáčová, and S. Hill for fruitful discussions. A portion of this work was performed at the National High Magnetic Field Laboratory, Tallahassee, FL, which is supported by NSF Cooperative Agreement No. DMR-0084173, by the State of Florida, and by the DOE. S.A.Z. acknowledges the support from the NHMFL through the VSP No. 1382. The Monte Carlo simulation was carried out on SGI Altrix 3700Bx2 at the Supercomputer Center, ISSP, University of Tokyo. A.P.F and N.F.O., Jr. are grateful for support from CNPq and FAPESP (Brazil).

[1] F.D.M. Haldane, Phys. Lett. 93A, 464 (1983); Phys. Rev. Lett. 50, 1153 (1983).

[2] R. Botet et al., Phys. Rev B 28, 3914 (1983); M.P. Nightingale and H.W.J. Blote, Phys. Rev. B 33, 659 (1986); T. Sakai and M. Takahashi, Phys. Rev. B 42, 1090(R) (1990).

[3] O. Golinelli et al., Phys. Rev. B 46, 10854 (1992).

[4] T. Sakai and M. Takahashi, Phys. Rev. B 42, 4537 (1990).

[5] R. Silberglitt and J.B. Torrance, Jr., Phys. Rev. B 2, 772 (1970).

[6] S.T. Chiu-Tsao et al., Phys. Rev. B 12, 1819 (1975).

[7] N. Papanicolaou and P.N. Spathis, Phys. Rev. B 52, 16001 (1995).

[8] M. Orendáč et al., Phys. Rev. B 52, 3435 (1995).

[9] N. Papanicolaou et al., Phys. Rev. B 56, 8786 (1997).

[10] A.K. Kolezhuk and H.J. Mikeska, Prog. Theor. Phys. Suppl. 145, 85 (2002); Phys. Rev. B 65, 014413 (2001).

[11] C. D. Batista and G. Ortiz, Phys. Rev. Lett. 86, 1082 (2001); Adv. Phys. 53, 1 (2004).

[12] I. Affleck, Phys. Rev. B 43, 3215 (1991).

[13] T. Giamarchi and A.M. Tsvelik, Phys. Rev. B 59, 11398 (1999).

[14] T. Nikuni et al., Phys. Rev. Lett. 84, 5868 (2000).

[15] A. Paduan-Filho et al., J. Chem. Phys. 74, 4103 (1981).

[16] A. Paduan-Filho et al., Phys. Rev. B 69, 020405(R) (2004).

[17] V.S. Zapf et al., Phys. Rev. Lett. 96, 077204 (2006).

[18] B. R. Cooper et al., Phys. Rev. 127, 57 (1962).

[19] S.A. Zvyagin et al., Physica B 346-347, 1 (2004).

[20] A minor impurity phase was detected in some ESR spectra. A1though the origin of this phase is not clear at the moment, we speculate that it is most likely originated from a superficial layer of DTN-crystals attacked by the GE-varnish solvent, which was used to fix the sample inside the sample-holder.

[21] With accuracy better than $\pm 0.5 \%$. For further references: the anisotropy constant $D$ is calculated with accuracy better than $\pm 3 \%$; the accuracy of estimation of exchange parameters is basically determined by accuracy of estimating the upper critical field $H_{c 2}$ and by accuracy of neutron scattering measurements [17], which is better than $\pm 10 \%$.

[22] R.F. Wallis et al., Phys. Rev. B 52, R3828 (1995).

[23] R. Lai et al., Phys. Rev. B 54, R12665 (1996).

[24] S.A. Zvyagin et al., Czech. J. Phys. 46, 1937 (1996); M. Orendáč et al., Phys. Rev. B 60, 4170 (1999). 